\title{
Laser-Induced Interfacial Spallation for Controllable and Versatile Delamination of Flexible Electronics
}

Jing Bian ${ }^{\dagger,,, \S}$, Furong Chen ${ }^{\dagger, \ddagger}$, Biao Yang ${ }^{\dagger, \ddagger}$, Jinlong Hu ${ }^{\dagger, \ddagger}$, Ningning Sun ${ }^{\dagger, \ddagger}$, Dong Y $e^{\dagger, \ddagger}$, Yongqing Duan $^{\dagger, \ddagger}$, Zhouping Yin ${ }^{\dagger, \ddagger}$, YongAn Huang ${ }^{*,+,}$

${ }^{\dagger}$ State Key Laboratory of Digital Manufacturing Equipment and Technology, Huazhong University of Science and Technology, Wuhan 430074, China

${ }^{\ddagger}$ Flexible Electronics Research Center, Huazhong University of Science and Technology, Wuhan 430074, China

$\S$ College of Electronic and Optical Engineering \& College of Microelectronics, Nanjing University of Posts and Telecommunications, Nanjing, 210023, China

*E-mail: yahuang@hust.edu.cn

This supporting information file includes:

Figures S1-S12, Text 1-2 

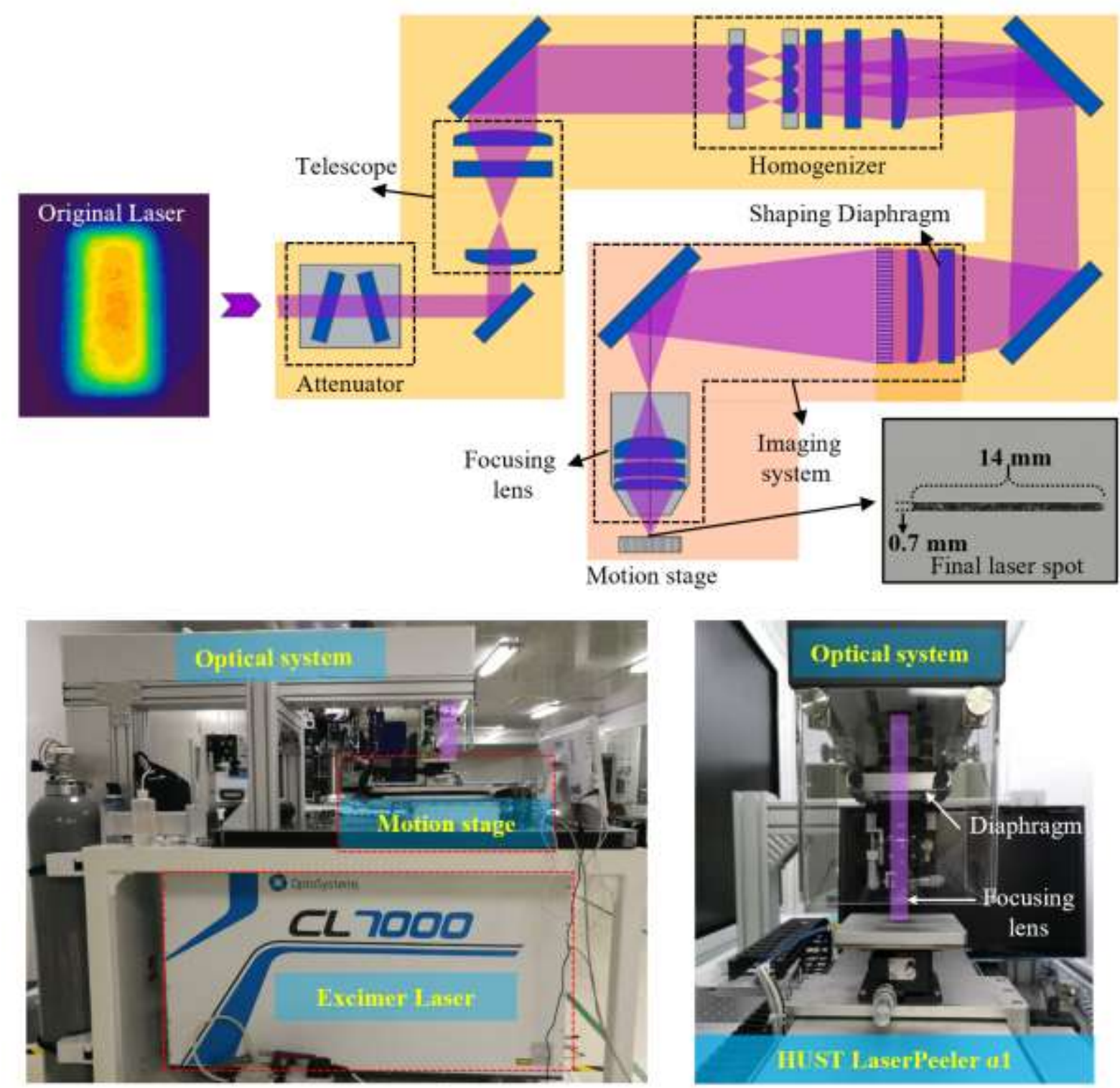

Figure S1 Experimental platform of LLO involved in this study. A pulsed laser (XeCl excimer laser, OptoSystems CL7020) with a wavelength of $308 \mathrm{~nm}$, pulse duration of $20 \mathrm{~ns}$, and the maximum repetition rate of $20 \mathrm{~Hz}$ is used as the light source. The laser beam passes through the attenuator to adjust the output energy of the laser beam (10\% 90\%). Nonuniformity of the laser beam is controlled less than $\pm 5 \%$ after using homogenizer and the beam is shaped into narrow rectangular by shaping telescope. Finally, a line-shaped beam profile $(14 \mathrm{~mm} \times 0.7 \mathrm{~mm})$ is obtained on the focal plane by using the diaphragm and focusing lens. By adjusting the height of the motion stage, the focal position of the laser spot can be located at the interface between PI film and glass substrate. By moving the stage at a certain speed, the LLO process can be achieved. 

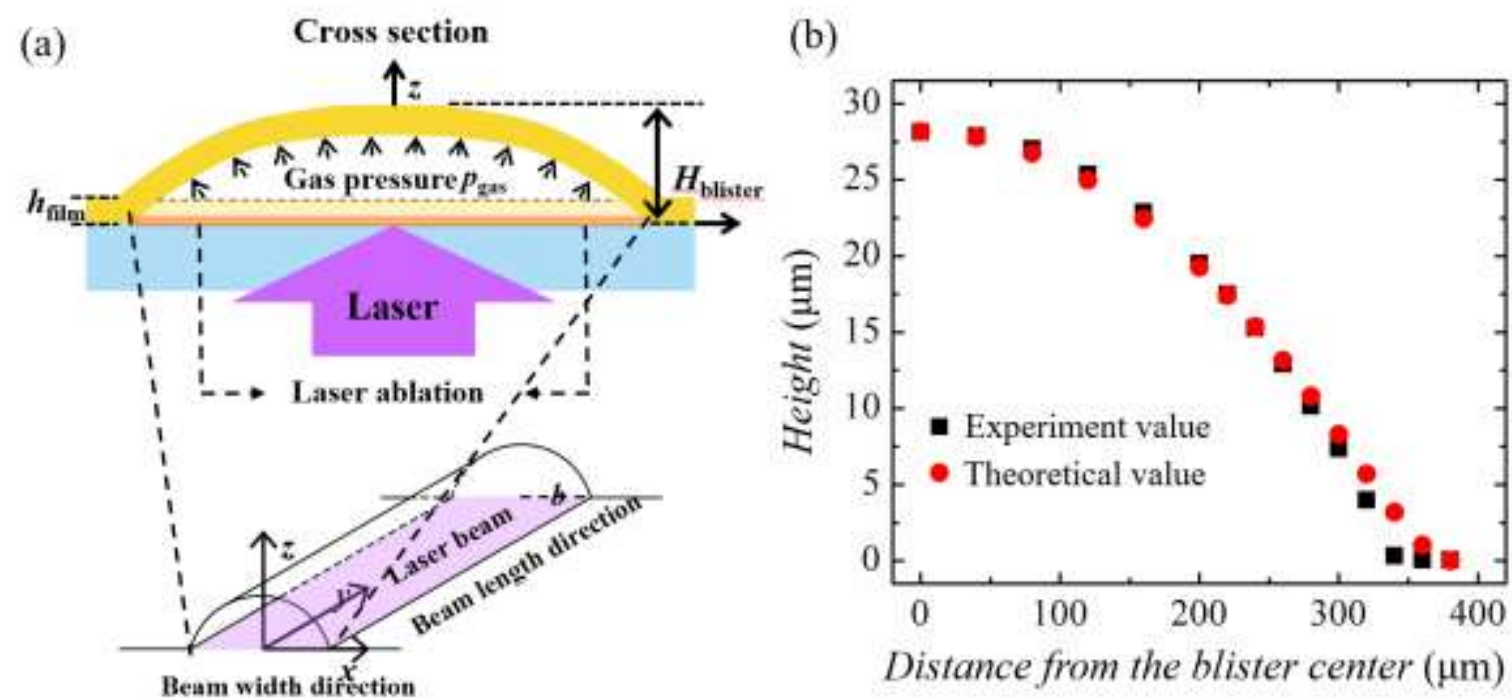

Figure S2 (a) Schematic diagram of a blister formed by laser irradiations. The semi-cylindrical geometry of the investigated blister aligned with the y-axis. (b) Comparison of the theoretical value and experimental value of the blister profile $(4.8-\mu \mathrm{m}$ thick film) under 50 times of irradiations with low fluence $\left(94 \mathrm{~mJ} / \mathrm{cm}^{2}\right)$. 

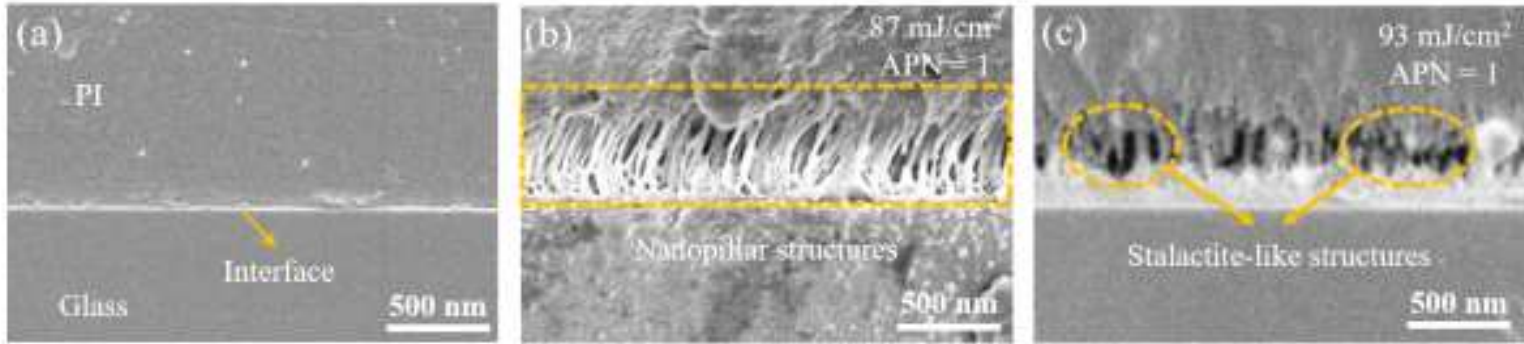

Figure S3 Microscopic observations of PI-glass interface before and after single laser irradiation. (a) before laser irradiation. (b) 87 $\mathrm{mJ} / \mathrm{cm}^{2}$; (c) $93 \mathrm{~mJ} / \mathrm{cm}^{2}$ 


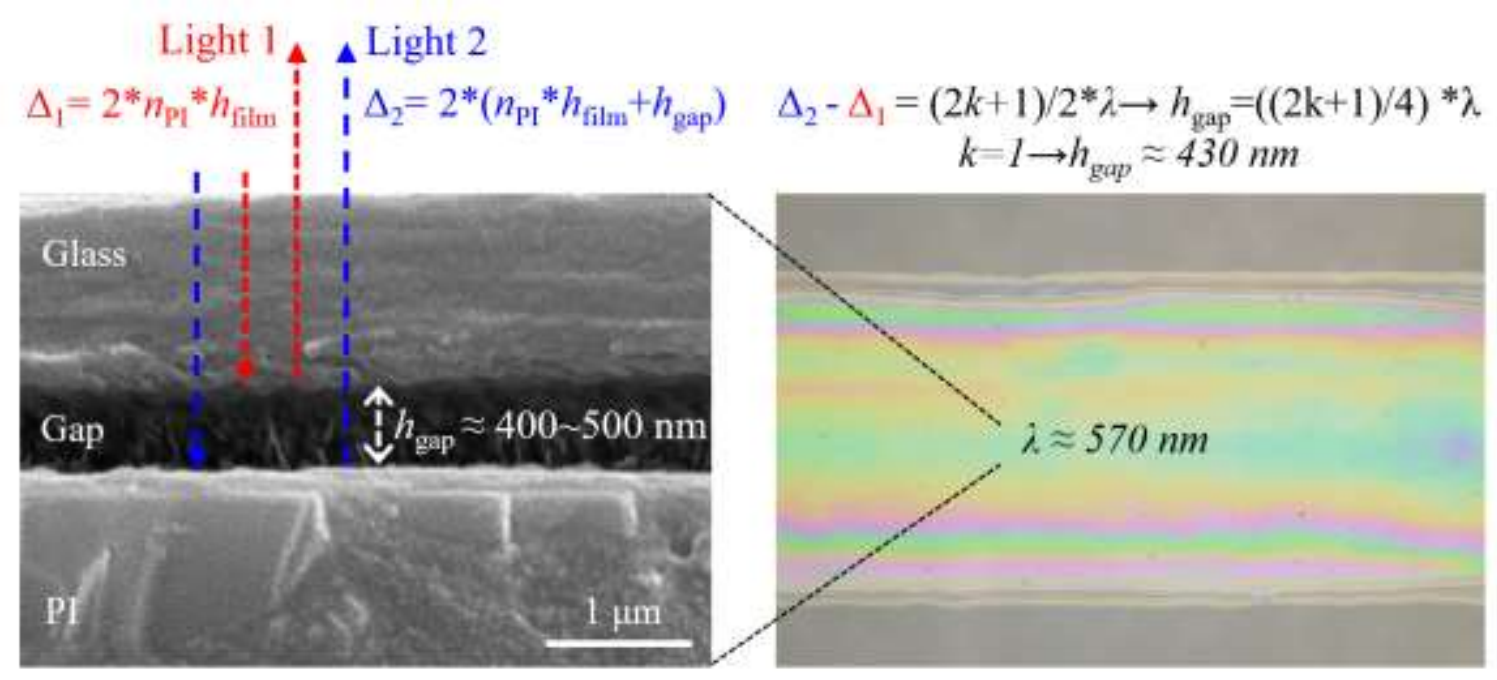

Figure S4 Illustration of the equal thickness interference of the two reflected light from the upper and lower surfaces of the gap led to the appearance of the bright stripe. The optical path difference between two reflected light should be $(2 \mathrm{k}+1) / 2$ times of the wavelength of the interference light. Accordingly, the height of the gap can be estimated. 


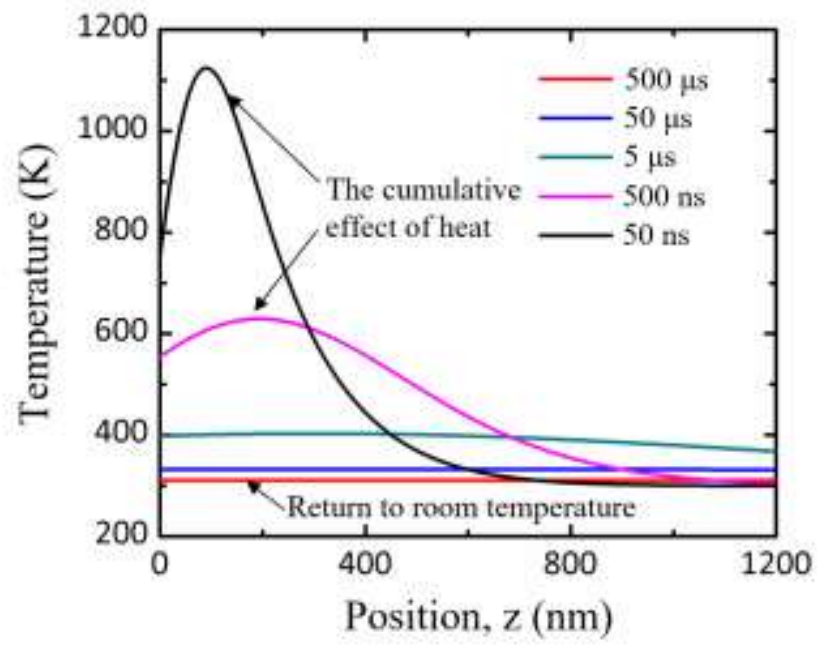

Figure S5 Theoretical calculations of temperature distributions of the interfacial PI with different cooling times after single laser irradiation $\left(70 \mathrm{~mJ} / \mathrm{cm}^{2}\right)$. 


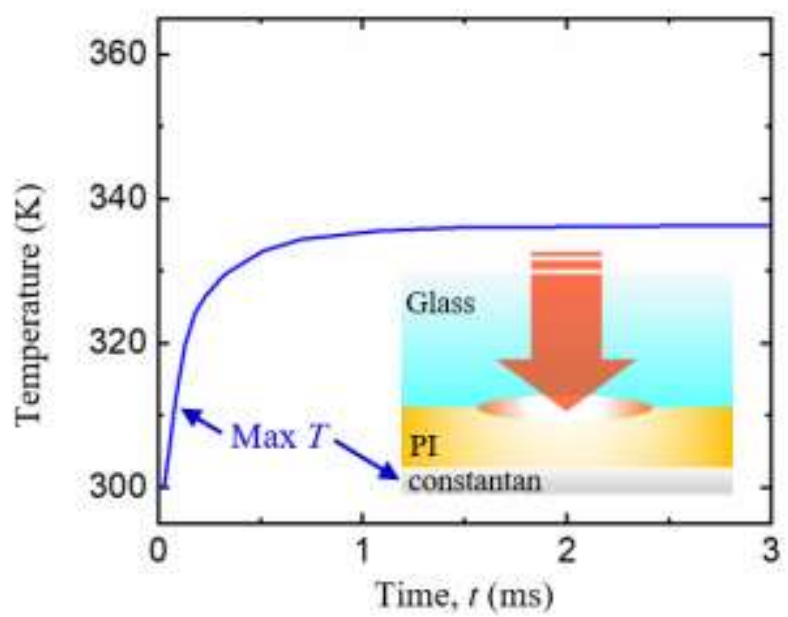

Figure S6 Calculated maximum temperature in the constantan layer varies with time under the laser irradiation. Glass thickness: 10 $\mu \mathrm{m}$; Polyimide layer thickness: $8 \mu \mathrm{m}$; Constantan thickness: $200 \mathrm{~nm}$; laser fluence: $100 \mathrm{~mJ} / \mathrm{cm}^{2}$. 


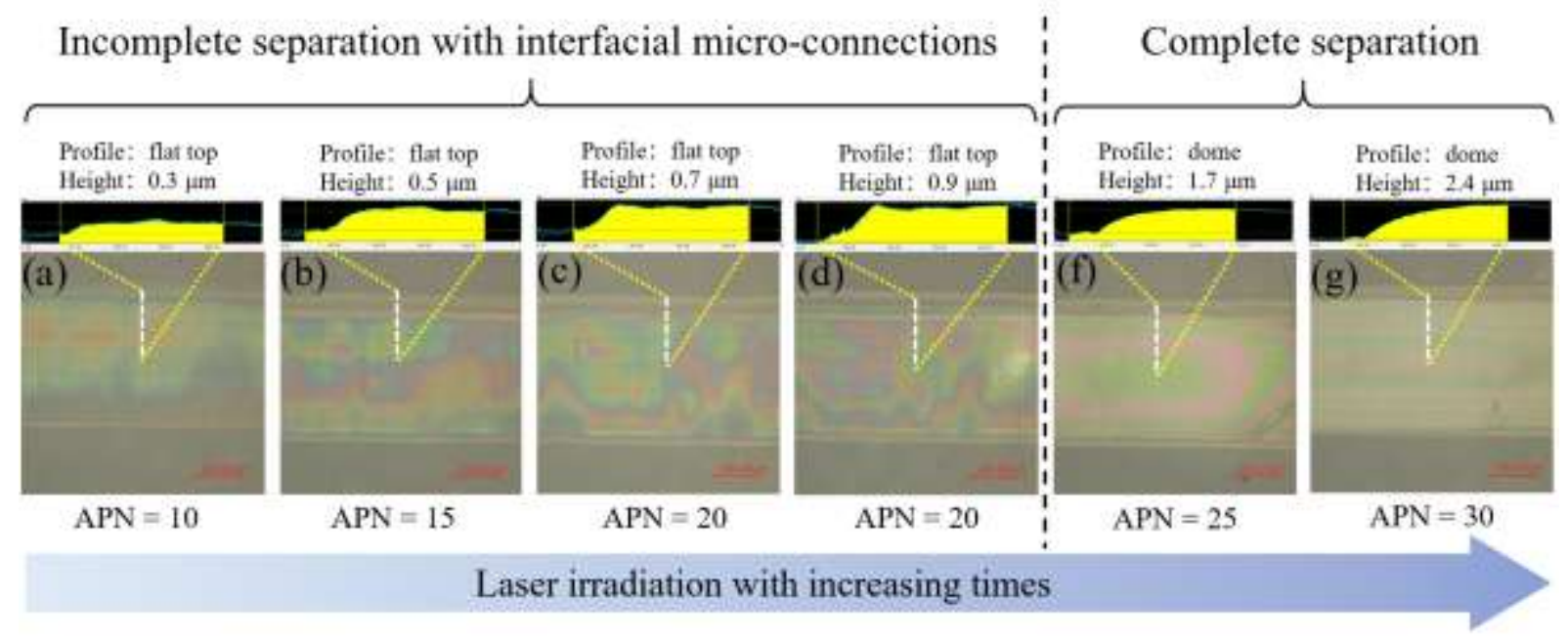

Figure S7 Optical observations of the irradiated PI film with increasing irradiation times through a laser scanning confocal microscope.

When the irradiation number is insufficient $(\mathrm{APN}<25)$, the irradiated region had a flat top profile, indicating the incomplete separation with interfacial micro-connections. Once the irradiation number is sufficient (APN > 25), the disappearance of the interfacial micro-connections made the profile of the irradiated region change from flat top to dome due to the trapped gas products. 
(a)

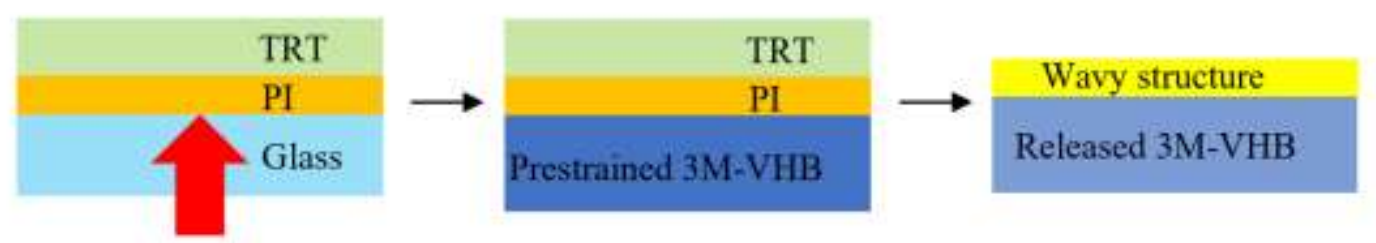

(b)

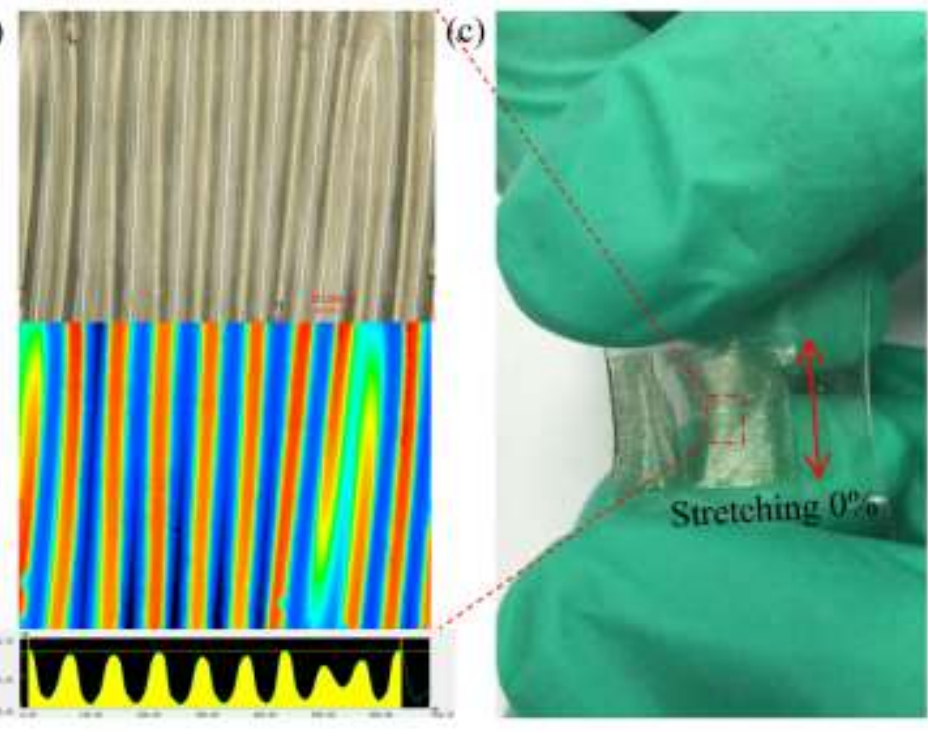

(d)

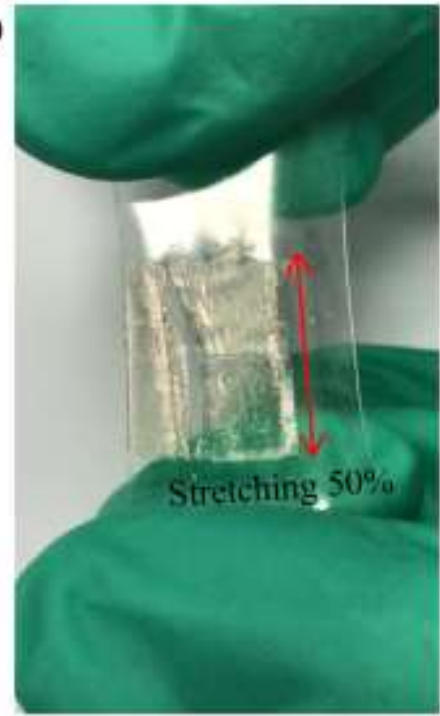

Figure S8 (a) Schematic illustrations of the fabrication process of a wavelike PI film for stretchable electronics. The ultrathin PI film was released from the glass through LIIS process and transferred onto a prestrained elastomeric tape (3M-VHB). The wavy structures were formed by releasing the prestrained elastomer. (b) Optical observations of the formed wavy structures times through a laser scanning confocal microscope. (c-d) Demonstration of the stretchability of the wavelike PI film. 


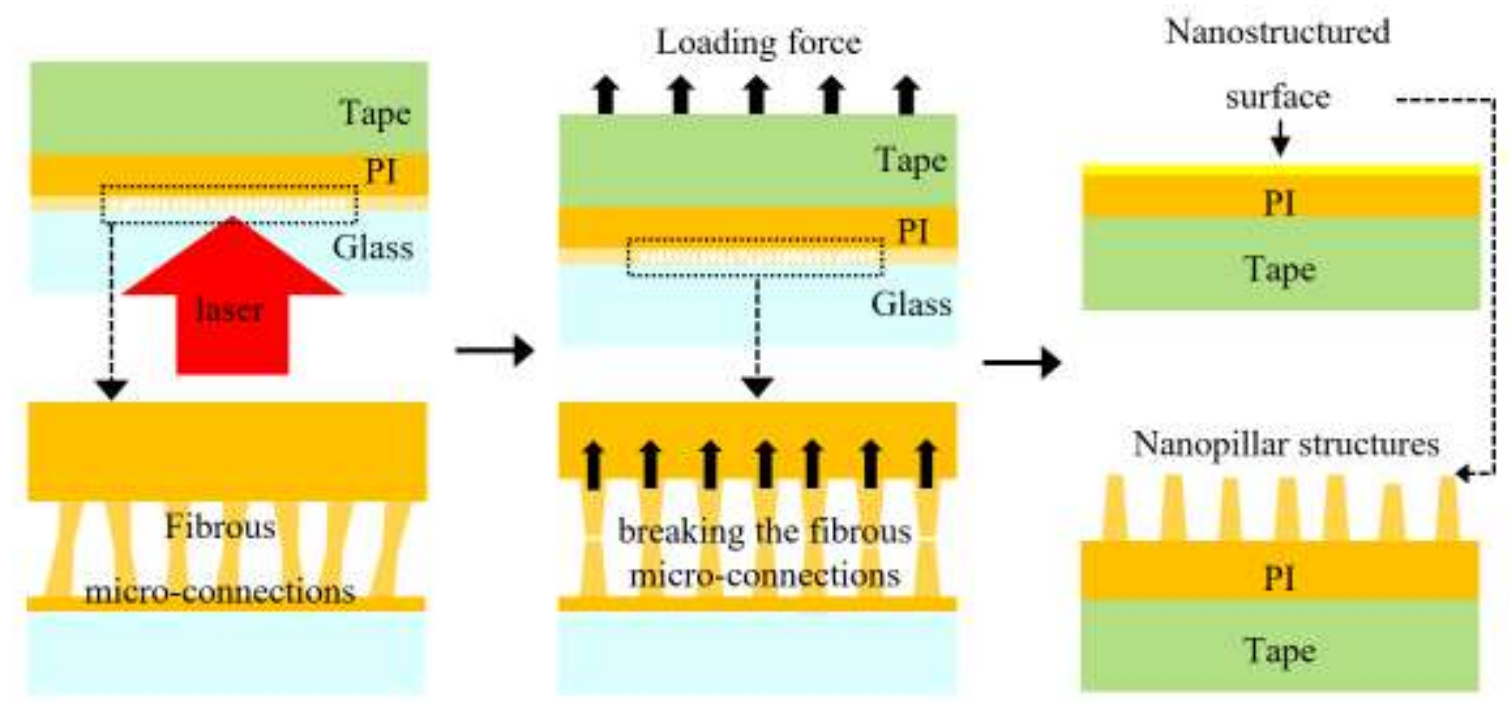

Figure S9 Schematic descriptions of the fabrication process of the nanopillar structures on the released PI film through LIIS. 
Arrow 1
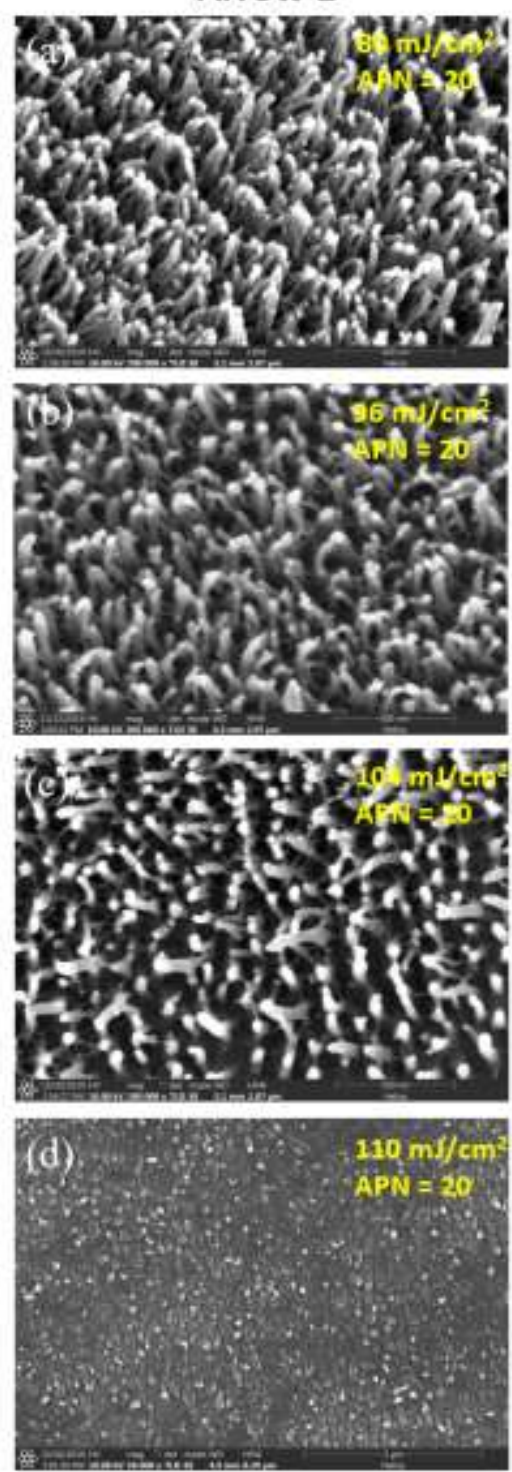

Arrow 2
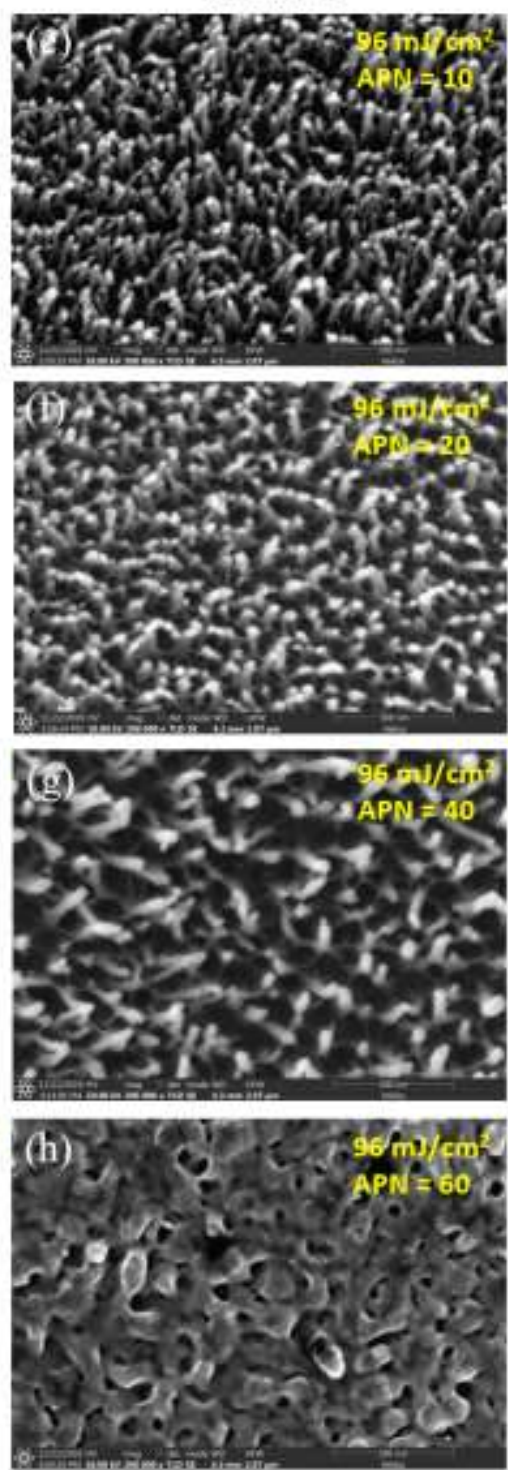

Arrow 3
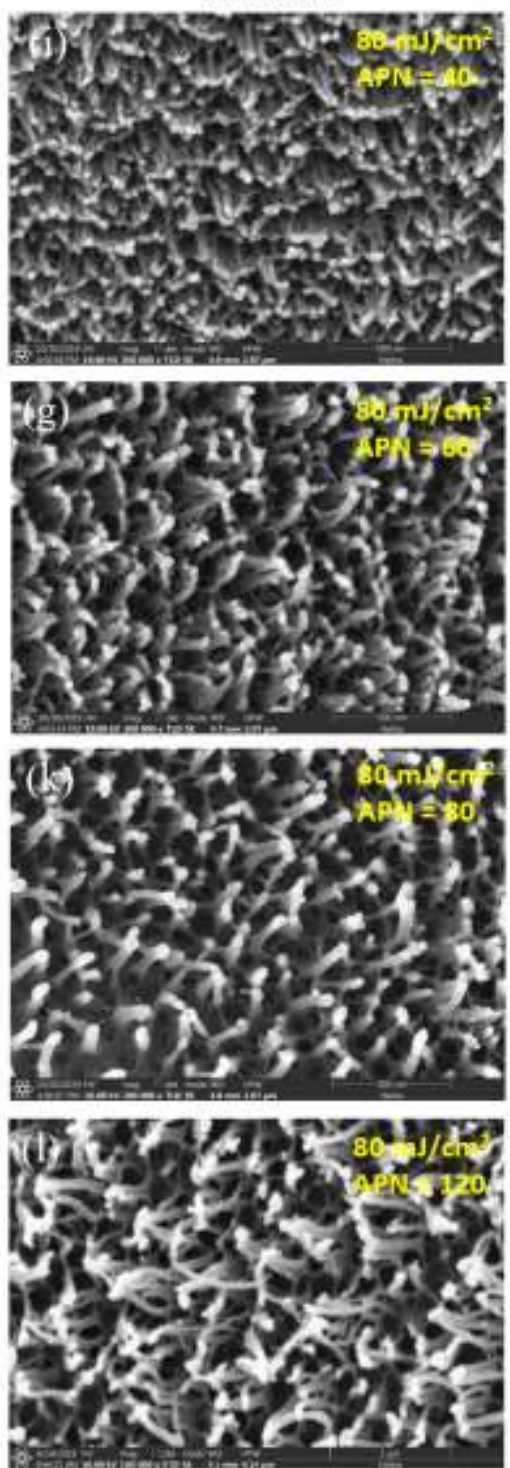

Figure S10 After the mechanical peeling process, the observed surface morphologies of the PI films with different sets of laser

parameters: (1) fixing APN $=20$, increasing the laser fluence (arrow 1 in Figure 5a); (2) fixing the laser fluence $\left(F_{\text {exp }}=96 \mathrm{~mJ} / \mathrm{cm}^{2}\right.$,

corresponding to $F_{\text {cal }}=58 \mathrm{~mJ} / \mathrm{cm}^{2}$ ), increasing the APN (arrow 2 in Figure 5a); and (3) fixing the laser fluence $\left(F_{\exp }=80 \mathrm{~mJ} / \mathrm{cm}^{2}\right.$,

corresponding to $F_{\text {cal }}=50 \mathrm{~mJ} / \mathrm{cm}^{2}$ ), increasing the APN (arrow 3 in Figure 5a). Only the samples with process parameters in the yellow

region of Figure 5a have nanostructured surfaces, while the samples with process parameters in the red region have relatively smooth

surfaces. However, the PI films with process parameters in the green region cannot be peeled off by adhesive tapes. Moreover, as the

process parameters were close to the green region of Figure 5a, the obtained nanopillar structures are relatively dense and short. In

contrast, once the process parameters were closer to the red region of Figure 5a, the nanopillar structures become sparse and longer in

height. 
(a)

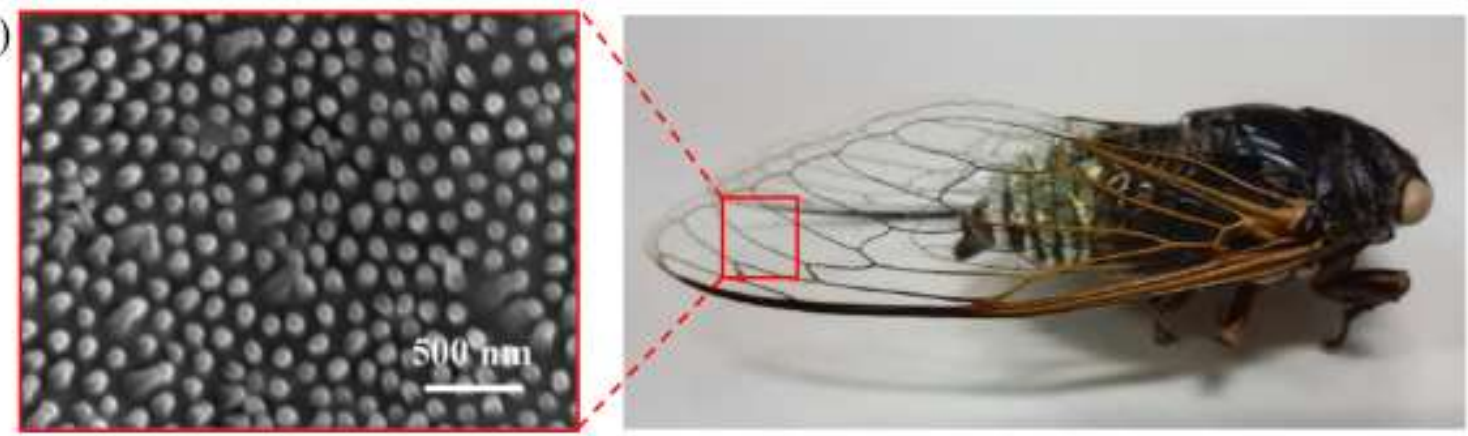

(b)

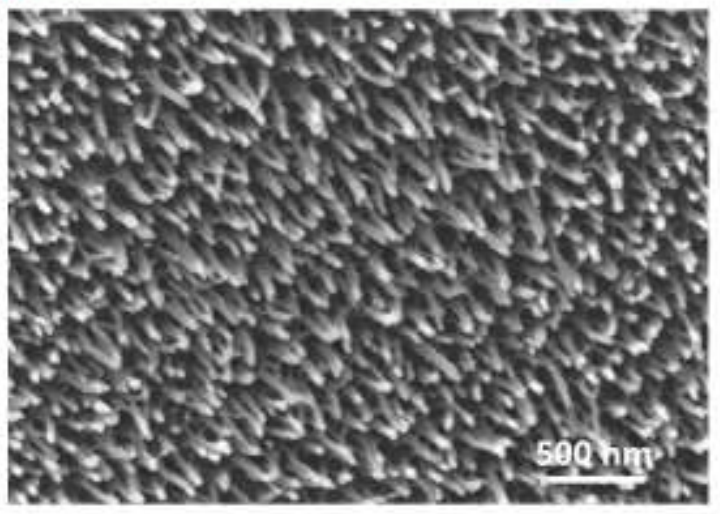

(c)

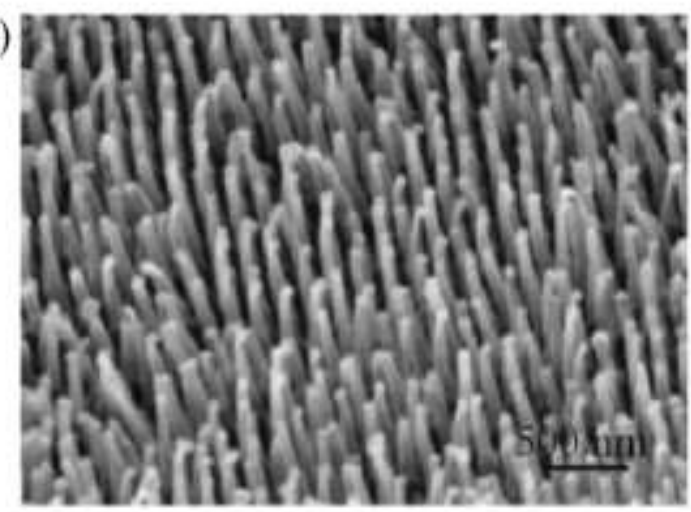

Figure S11 Nanopillar surface prepared by the LIIS process is similar to the surface of the cicada wing, and its simulation effect is not inferior to the nanopillar surface prepared using a bio-template (the cicada wing). (a) SEM image of the nanopillar cicada wing surface, obtained from [Xie H., Huang H.-X., Peng Y.-J.. Rapid fabrication of bio-inspired nanostructure with hydrophobicity and antireflectivity on polystyrene surface replicating from cicada wings. Nanoscale, 2017, 9(33): 11951-11958.]. (b) PI films with cicada wing-inspired nanopillar surfaces fabricated by the LIIS process. (c) Artificial nanostructures using the cicada wing as a bio-template, obtained from [Xie G., Zhang G., Lin F., et al. The fabrication of subwavelength anti-reflective nanostructures using a bio-template. Nanotechnology, 2008, 19(9): 095605.]. 
(a) A gradient refractive index
between the PI film and air An abrupt change in refractive index

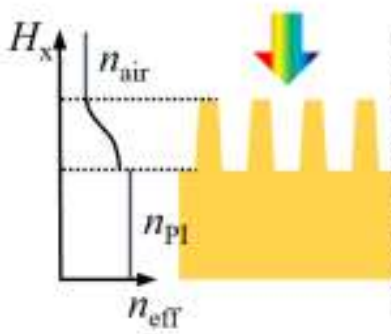

Nanostructured surface

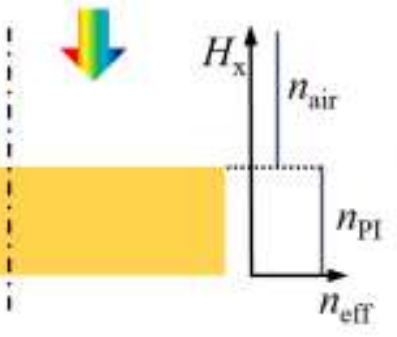

Smooth surface (b)

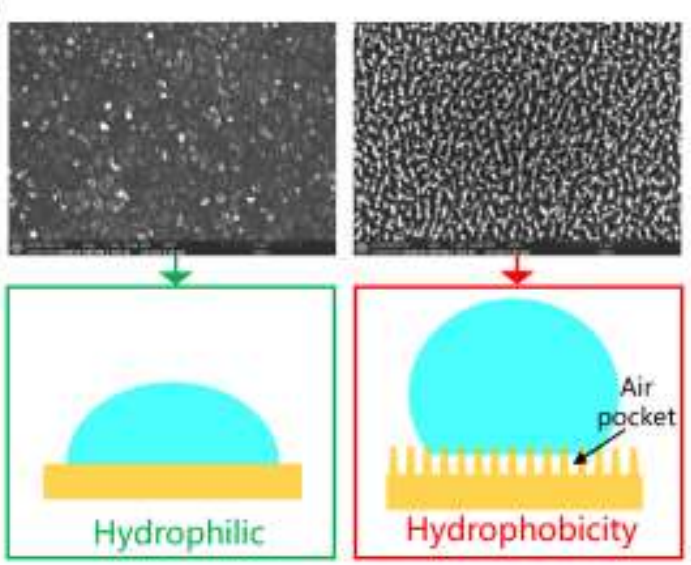

Figure S12 (a) Schematic description of the mechanism of optical trapping of nanostructured surfaces, as sub-wavelength nanostructures can be approximated as a set of multiple layers with a gradient refractive index between the PI substrate and air. (b)

Schematic description of the mechanism of hydrophobicity of nanopillars. 
Text 1: Parameters used for modeling of the ablation process involved in LIIS

Parameters used in calculations

\begin{tabular}{ccc}
\hline Parameter & Value & Reference \\
\hline Activation energy $E_{\mathrm{b}}[\mathrm{eV}]$ & 0.7 & {$[1]$} \\
Pre-exponential factor $k_{0}$ & $2.67 \times 10^{12}$ & {$[1]$} \\
Volumetric reaction & 2000 & $\mathrm{~L}={ }^{12} H_{\mathrm{b}} N_{0}$ \\
Enthalpy $L\left[\mathrm{~J} / \mathrm{cm}^{3}\right]$ & & \\
Reaction enthalpy per bond $H_{\mathrm{b}}$ & 1.4 & {$[2]$} \\
[eV] & & \\
Number density of bonds $N_{0}$ & $8.95 \times 10^{21}$ & {$[3]$} \\
Density of PI $\rho\left[\mathrm{g} / \mathrm{cm}^{-3}\right]$ & 1.42 & {$[4]$} \\
Density of glass $\rho\left[\mathrm{g} / \mathrm{cm}{ }^{-3}\right]$ & 2.37 & {$[5]$} \\
Specific heat of PI $C_{\mathrm{p}}[\mathrm{J} / \mathrm{g} \cdot \mathrm{K}]$ & $2.55-1.59 \times \exp \left[\left(T_{0}-T\right) / 460\right]$ \\
Specific heat of glass $C_{\mathrm{p}}[\mathrm{J} / \mathrm{g} \cdot \mathrm{K}]$ & $1.53-0.79 \times \exp \left[\left(T_{0}-T\right) / 638\right]$ & {$[5]$} \\
Thermal conductivity & $1.55 \times 10^{-3}\left(T / T_{0}\right)^{0.28}$ & \\
of PI $\kappa[\mathrm{W} / \mathrm{cm} \cdot \mathrm{K}]$ & & $44]$ \\
Thermal conductivity & & \\
of glass $\kappa[\mathrm{W} / \mathrm{cm} \cdot \mathrm{K}]$ & &
\end{tabular}

\section{Reference:}

[1] Bityurin N., Malyshev A. Bulk photothermal model for laser ablation of polymers by nanosecond and subpicosecond pulses. Journal of Applied Physics, 2002, 92(1): 605-613.

[2] Arnold N, Bityurin N. Model for laser-induced thermal degradation and ablation of polymers. Applied Physics A, 1999, 68(6): 615-625.

[3] Du Pont Product Bulletin. Kapton-polyimide film, summary of properties, 1987.

[4] Kim D.-H., Kim B.-K., Kim H.J., et al. Crystallization of amorphous silicon thin-film on glass substrate preheated at $650 \mathrm{C}$ using Xe arc flash of $400 \mu$ s. Thin Solid Films, 2012, 520(21): 6581-6588.

[5] Bessonov M. Polyimides--thermally stable polymers. Consultants Bureau, 1987. 
Text 2: Calculations of the amount of gas products generated by laser irradiations based on the optical measurements of the deformation of the irradiated PI film

After a certain number of laser irradiations, a PI film can be delaminated from the glass substrate and deformed into a semi-cylindrical blister (because the laser beam is line-shape) due to its internal gas products. As shown in Figure 2a, the profile of a blister can be measured by a laser scanning confocal microscope, therefore, the volume of gas products $V_{\mathrm{g}}$ can be obtained. For a specific measured profile, the inside gas pressure $P_{\mathrm{g}}$ can be derived according to a linear elastic model, which has been established to simulate the equilibrium displacements of the deformed PI film (the blister), as shown in Figure S2a. A semi-cylindrical blister aligned with the y-axis can be described as a thin plate with clamped boundaries and subjected to constant gas pressure. As the line-shape laser beam makes the profile of the blister not sensitive to its length direction (y-axis), thus, a governing equation for large deflections of a thin plate under normal uniform pressure $p_{\text {gas }}$ in $2 \mathrm{D}$ can be established and solved.

Here, we present detailed solutions for the displacements of a semi-cylindrical blister under uniform gas pressure $p=$ $p_{\text {gas. }}$ The von Karman plate theory for large plate deflections are

$$
D \nabla^{2} \nabla^{2} w=p+\sigma_{x} h \frac{\partial^{2} w}{\partial x^{2}}+\sigma_{y} h \frac{\partial^{2} w}{\partial y^{2}}+2 \tau_{x y} h \frac{\partial^{2} w}{\partial x \partial y}
$$

The membrane stress $\sigma_{\mathrm{x}}, \sigma_{\mathrm{y}}$, and $\tau_{\mathrm{xy}}$ must be considered in the large deflection model. The length of the blister is much larger than the blister width, thus, an infinite length plate can be approximated. All unknowns are independent of $y$, and the equation is simplified as follows:

$$
\frac{d^{4} w}{d x^{4}}-T_{x} \frac{d^{2} w}{d x^{2}}=p
$$

Nondimensionalize this equation with the following substitutions: $\xi=\frac{x}{b} ; \omega=\frac{w}{h}$

$$
\frac{d^{4} \omega}{d \xi^{4}}-\frac{T_{x} b^{2}}{D} \frac{d^{2} \omega}{d \xi^{2}}=\frac{p b^{4}}{h D}
$$

Define the dimensionless membrane force $k$ and pressure $P$ by $\frac{T_{x} b^{2}}{D}=k^{2} ; P_{\text {nor }}=\frac{p b^{4}}{h D}$. The dimensionless governing equation:

$$
\frac{d^{4} \omega}{d \xi^{4}}-k^{2} \frac{d^{2} \omega}{d \xi^{2}}=P_{\text {nor }}
$$

The general solution of this kind of equation is given:

$$
\omega(\xi)=\frac{C 2 e^{k \xi}}{k^{2}}+\frac{C 1 e^{-k \xi}}{k^{2}}-\frac{1}{2} \frac{P \xi^{2}}{k^{2}}+C 3 \xi+C 4
$$


Boundary conditions of the clamped plate on both sides:

$$
\left.\omega\right|_{\xi=-1}=0 ;\left.\quad \omega\right|_{\xi=1}=0 ;\left.\quad \frac{d \omega}{d \xi}\right|_{\xi=-1}=0 ;\left.\quad \frac{d \omega}{d \xi}\right|_{\xi=1}=0
$$

Substitute the general solution into the boundary conditions:

$$
C 1=\frac{P}{k\left(e^{k}-e^{-k}\right)} ; C 2=\frac{P}{k\left(e^{k}-e^{-k}\right)} ; C 3=0 ; C 4=\frac{1}{2} \frac{\left(e^{k} k-e^{-k} k-2 e^{k}-2 e^{-k}\right) P}{k^{3}\left(e^{k}-e^{-k}\right)}
$$

The general solution turns into:

$$
\omega(\xi)=\frac{P e^{k \xi}}{k^{3}\left(e^{k}-e^{-k}\right)}+\frac{P e^{-k \xi}}{k^{3}\left(e^{k}-e^{-k}\right)}-\frac{1}{2} \frac{P \xi^{2}}{k^{2}}+\frac{1}{2} \frac{\left(e^{k} k-e^{-k} k-2 e^{k}-2 e^{-k}\right) P}{k^{3}\left(e^{k}-e^{-k}\right)}
$$

The relative displacement of the two boundaries is zero:

$$
\left.u_{x}\right|_{x=b}-\left.u_{x}\right|_{x=-b}=0
$$

From the strain-displacement relation and Hooke's law, the equation A.9 turns into:

$$
\left.u_{x}\right|_{x=b}-\left.u_{x}\right|_{x=-b}=\int_{-b}^{b} \frac{\partial u_{x}}{\partial x} d x=\int_{-b}^{b}\left[\frac{1}{E h}\left(T_{x}-\mu T_{y}\right)-\frac{1}{2}\left(\frac{\partial w}{\partial x}\right)^{2}\right] d x=0
$$

Simplify the A.10, one can get:

$$
T_{x}=\frac{E h}{2\left(1-\mu^{2}\right) \times 2 b} \int_{-b}^{b}\left(\frac{d w}{d x}\right)^{2} d x
$$

Nondimensionalize the A.11:

$$
\int_{-1}^{1}\left(\frac{d \omega}{d \xi}\right)^{2}=\frac{1}{3} k^{2}
$$

Substitute the A.8 into the A.11:

$$
\frac{1}{3} \frac{P^{2}\left(2 e^{4 k} k^{2}-9 e^{4 k} k+12 e^{4 k}-16 e^{2 k} k^{2}-24 e^{2 k}+2 k^{2}+9 k+12\right)}{k^{6}\left(e^{4 k}-2 e^{2 k}+1\right)}=\frac{1}{3} k^{2}
$$

Once the dimensionless pressure $P$ is determined, by solving the transcendental equation A.13, the dimensionless membrane force $k$ can be obtained. As a result, the $\omega(\xi)$ can be calculated.

By continuously adjusting the gas pressure used in the theoretical model, once the calculated profile perfectly matches the observed blister profile (e.g. Figure $\mathbf{S 2 b})$, the inside gas pressure $P_{\mathrm{g}}$ can be obtained $\left(P_{\mathrm{g}}=p_{\mathrm{gas}}\right)$. Finally, the amount of gas products $n_{\mathrm{g}}$ can be obtained based on the ideal gas law, $n_{\mathrm{g}}=P_{\mathrm{g}} V_{\mathrm{g}} / R T_{\text {room}}$. When the laser fluence is too low, single irradiation cannot form a measurable blister. In this case, multiple irradiations were adopted, and the calculated amount of gas products had been divided by the number of irradiations. Hence, even a tiny amount of created gas products can also be measured. 